\title{
Passive Cannabis Smoking Resulting in a Coma in a 16 Month Old Infant
} Ahemd Refat Ragab ${ }^{1,2 *}$ and Maha Khald Al-Mazroua ${ }^{2}$

${ }^{1}$ Department of Forensic Medicine and Clinical Toxicology, Mansoura University, Egypt

${ }^{2}$ Dammam Regional Poison Control Center, KSA, Egypt

\begin{abstract}
We report on 16-month-old infant who was admitted to hospital with severe neurological symptoms following passive inhalation of cannabis. To our knowledge and after deep surveying in the literature, we believe that this is one of the few reported clinical case of the acute severe toxicity after passive inhalation of cannabis smoke by an infant. Urine and serum THC (delta-9-tetrahydrocannabinol) $\Delta^{9}-\mathrm{THC}$ concentrations of 230 and $112 \mathrm{ng} / \mathrm{ml}$ were detected. This case report highlights the importance of considering cannabis ingestion in the differential diagnosis of infantile and toddler coma. Moreover, facing the increasing number of cannabis smokers such cases might be more prevalent and therefore physicians, health workers and parents should be alerted about the health hazards of cannabis substances abuse in the presence of children.
\end{abstract}

Keywords: Passive smoking; Cannabis; Infant; Coma

\section{Introduction}

Cannabis is the general name used for products made from the plant Cannabis sativa, such as marijuana, hash and hash oil. This plant contains over 400 chemicals, with about 60 creating its unique effects. The main mind-altering ingredient in cannabis is THC (delta9-tetrahydrocannabinol) ( $\Delta^{9}$-THC). It is mostly responsible for the changes in mood, thoughts, perceptions and behaviour when you use the drug. Cannabis works by entering your bloodstream, and then travelling to specific cannabis receptors in the brain, where it is activated [1].

Unlike most other drugs of abuse, marijuana and other cannabinoid products are commonly exposed by smoking, a process which releases sidestream smoke to the user's immediate surroundings. It is important to keep in mind that several factors determine the amount of cannabinoids that may be exposed by passive inhalation. The exposure to a nonsmoking individual is affected by the amount of sidestream smoke released; the concentration of $\Delta^{9}$-THC in the smoke; and the amount of smoke in the environment. Other important factors that affect passive exposure are the length of time an individual is exposed to the smoke; the type and size of the enclosure (room or vehicle); and the amount of ventilation during exposure [2].

The extent of illicit drug use has thus remained stable, but the estimated 15.5 million-38.6 million problem drug users (almost 12 per cent of illicit drug users), including those with drug dependence and drug-use disorders, remain a particular concern [3].

In infant and childhood period, one study [4], found that cannabis toxic effects are mainly discussed in various studies concerned with maternal prenatal exposure. These effects include neurobehavioral symptoms such as inattention, impulsivity, increased externalizing behavior and decreased cognitive performance with memory and learning impairments [5]. However, the direct exposure of infants to cannabis substances is discussed neither in clinical assessment nor in public health education. To our knowledge and after deep surveying the literature using Pubmed, Highwire, Google Search and Science Direct, we believe that this is one of the few reported clinical case of the acute severe toxicity after passive inhalation of cannabis smoke by an infant $[4,6,7]$.

The infant was admitted to hospital with severe neurological symptoms, following this toxic exposure. The significance of this topic is far beyond being one of the rare reported case of acute severe toxicities, but it also carries a significant social importance due to the increasing number of fathers who smoke near their children and thus is probably underdiagnosed as toxicology screens are uncommon by implemented in this age group; this fact underscores the importance of our report.

In this case report; we have directed the dangerous effects of passive cannabis inhalation and its liability to induce severe neurological manifestations in high level of $\Delta^{9}$-THC in blood and urine tests; to notify health worker, educator and parent about the dangerous health hazarads of passive cannabis smoking in their children's.

\section{Case Report}

A previously healthy 16 -months-old boy presented to the emergency department (ED) with the sudden onset of single attack of convulsion "tonic-clonic for one minute followed by short postictal fit" without a previous history of convulsive fits, followed by ataxia, unresponsiveness, with acute disturbed conscious level. The mother reported that her husband is cannabis smoker and her infant was sleeping in the same living room where her husband had been smoking cannabis cigarette (about 10 cigarettes for one and half hours with no remaining amount of smoked cannabis) without any suspected history for possibility of accidental cannabis intake by ingestion. She noticed that once the infant woke up he appeared ill, did not respond to verbal stimuli and refused to eat. The ER physician ruled out other causes of convulsive fits from history, clinical examination and basic clinical investigations. There was no indication of an infectious process, history of ingestion of toxic substance "involved cannabis"/drugs or head or other trauma. Pregnancy, delivery and early development were normal. He lived at home with his parents.

Examination revealed stupor with a Glasgow Coma Scale of 10; cranial nerves, muscle tone and deep tendon reflexes were normal; pupils were equal dilated ( $4.5 \mathrm{~mm}$ in diameter), sluggish reaction to

*Corresponding author: Ahemd Refat Ragab, Faculty of medicine, Department of Forensic Medicine and Clinical Toxicology, Mansoura University, Egypt, E-mail: ahmedrefat1973@yahoo.com

Received November 24, 2012; Accepted December 26, 2012; Published December 28, 2012

Citation: Ragab AR, Al-Mazroua MK (2012) Passive Cannabis Smoking Resulting in a Coma in a 16 Month Old Infant. J Clin Case Rep 2:237. doi:10.4172/2165 7920.1000237

Copyright: ( $) 2012$ Ragab AR, et al. This is an open-access article distributed under the terms of the Creative Commons Attribution License, which permits unrestricted use, distribution, and reproduction in any medium, provided the original author and source are credited. 
light (constricting to $3.5 \mathrm{~mm}$ with light), temperature $37.3^{\circ} \mathrm{C}$, blood pressure $98 / 40 \mathrm{mmHg}$, pulse 120 beats/min, respiratory rate 50 breaths/min and oxygen saturation $88 \%$ in room air "most commonly attributed to hypoventilation due to tachypnea that improved by supplemental oxygen. There was no evidence of head trauma. The rest of the examination was unremarkable. Laboratory evaluation revealed normal blood count, glucose and electrolytes and chest X-ray. An initial urine screen was positive for cannabis $\geq 135 \mathrm{ng} / \mathrm{mL}$. This was confirmed in a second analysis by immunochemistry as well as by Gas Chromatography/Mass Spectrometry (GC/MS) at the Dammam Regional Poison Control Center. Urine and serum THC concentrations of 230 and $112 \mathrm{ng} / \mathrm{ml}$ were detected "extremely above the normal level suspected in the passive cannabis smoker $(50 / 30 \mathrm{ng} / \mathrm{mL})$ ). He was admitted to the pediatric intensive care unit, where he received supportive treatment limited to intravenous fluids and nasal oxygen. His symptoms resolved rapidly within 24 hours. He was discharged after 48 hours.

\section{Discussion}

The clinical effects of THC are highly variable among individuals. In adolescents and adults, the acute effects of THC include dizziness, heart rate changes, blurred vision, altered sensorium, cognitive impairment, hallucinations and psychosis [8].

Despite the extensive knowledge on the effect of inhaled THC in adults, the reports on THC effects in infants are few and toxicity following passive inhalation of THC in this age group has been reported as the following clinical signs; tachycardia, conjunctival hyperemia, pallor, ataxia, nystagmus, fine tremor, lability of affect and stupor [911]. Signs and symptoms begin few hours after inhalation and usually resolve completely within 24 hours. Accidental cannabis ingestion has been reported to lead to coma in children [12].

In the present case, the infant was presented with altered state of consciousness after passive inhaling cannabis smoke. Considering that marked improvement was observed within 24 hours following admission under supportive treatment. Electrolytes' status, renal and hepatic functions were also considered and ruled out. However, a complete urine toxicology screen for all suspected substance of abuse "cannabis, opiate, benzodiazepines, barbiturates, cocaine, amphetamines and alcohol" were done. It was positive for 11-nor-9carbox $\left(\Delta^{9}\right.$-THC), the primary urinary metabolite of THC. A complete GC/MS drug screen was done with negative results. The parents' confirmation of lengthy and heavy exposure to cannabis smoke alongside the natural history of rapid improvement under supportive therapy, confirms the clinical and laboratory diagnosis of THC toxicity in this case.

This etiology should be taken into consideration in the differential diagnosis of altered states of consciousness, after excluding more common etiologies. Similarly, cannabinoids were detected in the urine of 5 volunteers who passively inhaled cannabis smoke for $30 \mathrm{~min}$ in a small closed car [13].

Subjective effects of THC after exposure to side-stream smoke were also reported [12]. A few reports speculated that children might be affected by passive cannabis inhalation, but clinical manifestations of such exposure were not reported $[12,13]$. One study examined the presence of cannabis metabolites in urine samples which were routinely obtained from asymptomatic infants. Low doses of cannabis metabolites were found in the urine of several children. However, the route and specific time of exposure, duration and clinical relevance were not addressed [14-16].
Notably, THC pharmacokinetic has not been studied in infants. THC, a lipophilic compound, tends to accumulate in fat tissues. Thus, it readily crosses the blood-brain barrier and reaches high levels in the brain. Its effect on infants might depend on respiratory parameters as well as body weight, total volume of distribution with regard for the relatively abundant fat tissues in infants and the susceptibility of the developing brain. The fact that the infant is smaller in body mass than an adult may hypothetically result in higher concentrations of THC followed by its noxious effects.

During the last years cannabis use has been increased, with an estimated 162 million (4\%) of the world's adults using it in 2004, a $10 \%$ increase on use in the mid 1990s [17]. These data suggest that cannabis abuse due to passive inhalation might be found even in young normative and well functioning families as opposed to other forms of abuse in which additional risk factors are usually identified.

As with other psychotropic substances, acute cannabis intoxication may last for 12-24 hours and cause a recognizable syndrome ('toxidrome') [9]. Although the marijuana toxidrome is easily recognizable among teenagers and adults, it is often unsuspected in young children. Cases of childhood coma after the ingestion of cannabis cookies have been previously reported $[18,19]$, indicating the importance of considering this possibility. The present report illustrates both the clinical consequences following childhood cannabis exposure and the importance of child protection considerations.

\section{Conclusions}

Therefore, it is of high importance to direct parents' attention to the possibility of abusive effects of passive inhalation of THC in children. As infants seem to be more vulnerable to THC toxicity, passive inhalation of the drug might create a serious health hazard for them. Medical staff should be aware of the clinical manifestations of cannabis intoxication since it is not routinely considered in medical assessment of infants under daily clinical settings. Discrepancy between the severe neurological presentation and the paucity of other findings in the physical examination and routine laboratory assessment should raise the diagnostic possibility of cannabis poisoning. In such cases, urine toxicology screen is imperative.

\section{References}

1. Bauman A, Phongsavan P (1999) Epidemiology of substance use in adolescence: Prevalence, trends and policy implications. Drug and Alcohol Dependence 55: 187-207.

2. Huestis MA, Cone EJ (2004) Relationship of Delta 9-tetrahydrocannabino concentrations in oral fluid and plasma after controlled administration of smoked cannabis. J Anal Toxicol 28: 394-399.

3. United Nations, Department of Economic and Social Affairs, Population Division, World Population Prospects (2010) Recent statistics and trend analysis of illicit drugs markets. World Drug Report 1: 8-14.

4. Amirav I, Luder A, Viner $Y$, Finkel M (2011) Decriminalization of cannabispotential risks for children? Acta Paediatr 100: 618-619.

5. Huizink AC, Mulder EJ (2006) Maternal smoking, drinking or cannabis use during pregnancy and neurobehavioral and cognitive functioning in human offspring. Neurosci Biobehav Rev 30: 24-41.

6. Cooper ZD, Haney M (2009) Actions of delta-9-tetrahydrocannabinol in cannabis: relation to use, abuse, dependence. Int Rev Psychiatry 21: 104-112.

7. Zarfin Y, Yefet E, Abozaid S, Nasser W, Mor T, et al. (2012) Infant with altered consciousness after cannabis passive inhalation. Child Abuse Negl 36: 81-83.

8. Reece AS (2009) Chronic toxicology of cannabis. Clin Toxicol (Phila) 47: 517 524 
Citation: Ragab AR, Al-Mazroua MK (2012) Passive Cannabis Smoking Resulting in a Coma in a 16 Month Old Infant. J Clin Case Rep 2:237. doi:10.4172/2165-7920.1000237

9. Bryson PD. Marijuana. In: Comprhensive review in toxicology for emergency clinicians. Washington, DC: Taylor \& Francis, 1996:531-534.

10. Blackstone M, Callahan J (2008) An unsteady walk in the park. Pediatr Emerg Care 24: 193-195.

11. Bonkowsky JL, Sarco D, Pomeroy SL (2005) Ataxia and shaking in a 2-year-old girl: acute marijuana intoxication presenting as seizure. Pediatr Emerg Care 21: $527-528$.

12. Macnab A, Anderson E, Susak L (1989) Ingestion of cannabis: a cause of coma in children. Pediatr Emerg Care 5: 238-239.

13. Morland J, Bugge A, Skuterud B, Steen A, Wethe GH, et al. (1985) Cannabinoids in blood and urine after passive inhalation of cannabis smoke. J Forensic Sci 30: 997-1002.
4. Cone EJ, Johnson RE (1986) Contact highs and urinary cannabinoid excretion after passive exposure to marijuana smoke. Clin Pharmacol Ther 40: 247-256.

15. Hicks JM, Morales A, Soldin SJ (1990) Drugs of abuse in a pediatric outpatient population. Clin Chem 36: 1256-1257.

16. Bhushan V, Ng S, Spiller D, Gang H, Inamdar S (1994) Detecting children's passive exposure to cocaine and marijuana. Am J Public Health 84: 675-676.

17. Hall W, Degenhardt L (2007) Prevalence and correlates of cannabis use in developed and developing countries. Curr Opin Psychiatry 20: 393-397.

18. Hall W, Solowij N (1998) Adverse effects of cannabis. Lancet 352: 1611-1616.

19. Appelboam A, Oades PJ (2006) Coma due to cannabis toxicity in an infant. Eur J Emerg Med 13: 177-179. 